\title{
A Comparative Study of Optical Concentrators for Visible Light Communications
}

Rahmat Mulyawan ${ }^{\mathrm{a}}$, Ariel Gomez ${ }^{\mathrm{a}}$, Hyunchae Chun ${ }^{\mathrm{a}}$, Sujan Rajbhandari ${ }^{\mathrm{b}}$, Pavlos P. Manousiadis ${ }^{\mathrm{c}}$, Dimali A. Vithanage ${ }^{\mathrm{c}}$, Grahame Faulkner $^{\mathrm{a}}$, Graham A. Turnbull ${ }^{\mathrm{c}}$, Ifor D.W. Samuel ${ }^{\mathrm{c}}$, Stephen Collins ${ }^{\mathrm{a}}$, Dominic O’Brien ${ }^{\mathrm{a}}$

a Department of Engineering Science, University of Oxford, Oxford, OX1 3PJ, United Kingdom; ' Department of Aerospace, Electrical and Electronic Engineering, Coventry University, Coventry, CV1 5FB, United Kingdom; ${ }^{\mathrm{c} S c h o o l}$ of Physics and Astronomy, University of St Andrews, St. Andrews, KY16 9SS, United Kingdom.

\begin{abstract}
Given the imminent radio frequency spectrum crunch, Visible Light Communication (VLC) is being proposed as an alternative wireless technology allowing for scalable connectivity to potentially millions of mobile and Internet-ofThings (IoT) devices. A VLC system uses a photo-detector (PD) receiver that converts the optically modulated light from a light source into a modulated electrical signal. The corresponding receiver electrical bandwidth is typically inversely proportional to the PD active area. Consequently, to construct a high-speed VLC link, the PD active area is often substantially reduced and an optical concentrator is used to enhance the receiver collection area. However, to achieve high concentrating factor, the link field-of-view (FOV) needs to be narrow due to the étendue conservation in linear passive optical systems. This paper studies a Fluorescent Concentrator (FC) that breaks this étendue conservation. The FC is not only based on reflective and refractive principles but also makes use of fluorescence process. A comparison between the FC and conventional optical concentrators, namely Compound Parabolic Concentrator (CPC) is also investigated. The trade-off between received signal strength and incoming link angle is demonstrated over $60^{\circ}$ coverage. Experimental results show that performance degradation as the link angle increases using FC-based receivers is significantly lower than for conventional CPC.
\end{abstract}

Keywords: optical concentrator, étendue, field-of-view, visible light communication

\section{INTRODUCTION}

Visible Light Communication (VLC) is a promising technology to solve the spectrum crunch in future wireless communication $^{1}$, partly because in the last decade there has been a surge of interest in solid-state lighting as a more energy-efficient technology to replace conventional lighting ${ }^{2}$. The performance of a VLC receiver can be improved by increasing signal strength received by increasing the area of the detector in the receiver. In this case, small photodetectors are preferable because their smaller capacitance means that they have a higher bandwidth (BW). The signal falling on these small photodetectors is often increased by using an optical element to concentrate light from a larger area onto these small photodetectors, as illustrated in Figure 1.
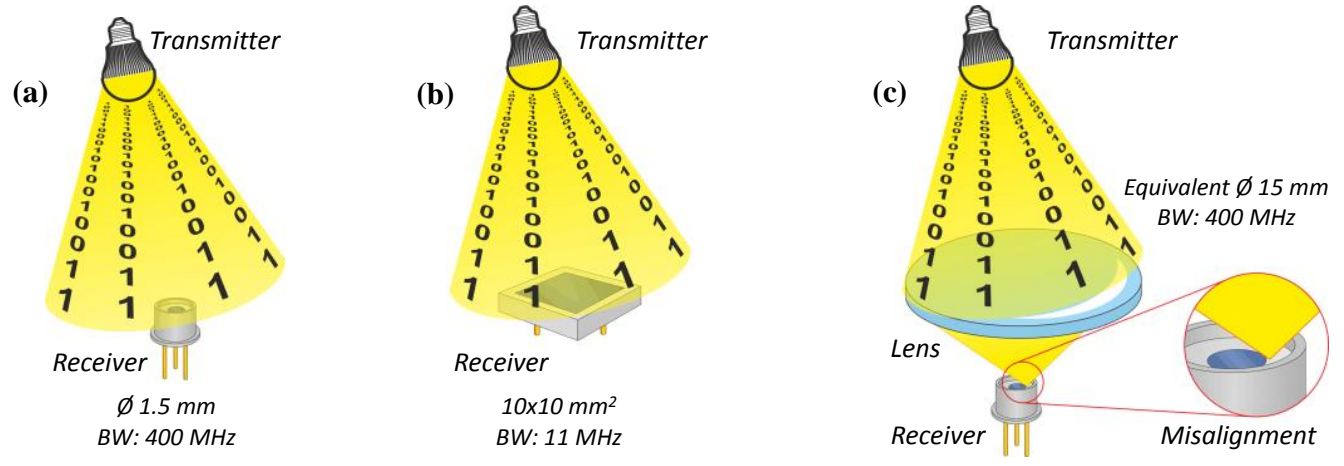

Figure 1. Illustration of the trade-off between the receiver active area and the BW: (a) small area with high BW receiver (b) large area with low BW receiver (c) large area with high BW receiver using a lens but prone to misalignment 
However, the gain of conventional optical concentrators is limited by étendue, a geometric property which indicates the flux gathering capability of the optical system. Étendue is conserved as light travels through an optical system where it undergoes reflections or refractions. Conservation of étendue means that the gain of this optical concentrator can only be increased at the expense of decreasing the receiver's field of view (FOV) ${ }^{3}$.

The problems arising from the conservation of étendue can be avoided by using an optical concentrator which not only relies upon the two processes, reflection and refraction. Recently, it has been suggested that fluorescence, an optical process that does not conserve étendue, could be used to create a wide field of view optical concentrator for VLC ${ }^{4-7}$

This paper studies a Fluorescent Concentrator (FC) that breaks étendue conservation, and compare its performance with a conventional (étendue-limited) concentrator, the Compound Parabolic Concentrator (CPC). The text is divided as follows: section 2 describes the working principles of both CPC and FC. Section 3 introduces an experimental study. The experimental results and discussion are presented in section 4. Finally, section 5 shows the conclusions.

\section{CONCENTRATOR PRINCIPLES}

\subsection{Compound Parabolic Concentrator (CPC)}

The CPC is a concentrator with a cross section of two parabolic sections such that the focus of each section is in the bottom corner of the other. Light incident on the entrance aperture reaches the exit plane after reflected on its side walls. The CPC can be made as a hollow conical shape with reflecting material or as a solid paraboloid made from dielectric material. The paraboloid CPC has a better concentrating factor (gain) than the hollow one ${ }^{8}$ and is more widely used in VLC applications.



(a)

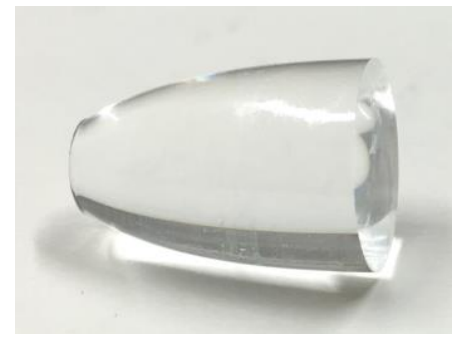

(b)

Figure 2. (a) A schematic diagram of the physical processes in a CPC; (b) A photograph of a commercial CPC made from B270 glass $(n=1.51)$.

Figure 2.a. illustrates how the incoming rays from free space enter the input aperture of a CPC made from dielectric material. The rays are refracted and retained by Total Internal Reflection (TIR) until they reach the output aperture, where a photo-detector (PD) is coupled. The relationship between the maximum gain $G_{\text {max }}$ and the acceptance angle $\theta_{\max }$ is given by:

$$
G_{\text {max }}=\frac{n^{2}}{\sin ^{2} \theta_{\max }}
$$

where $n$ is the refractive index of the CPC material. Since the CPC is designed to guide the light at certain acceptance angle $\theta_{\max }$, any light coming with a larger angle than $\theta_{\max }$ is not able to reach the output aperture, thus cannot be detected by the PD.

The relation of gain and FOV from Equation 1 for a typical CPC is shown in Figure 3. This figure suggests that, although the CPC can achieve high gain, the FOV of the CPC will be consequently narrow, thus limiting the practical applications. In other hand, a CPC with a wide FOV of 60 degrees will only have a maximum gain of approximately 3. 


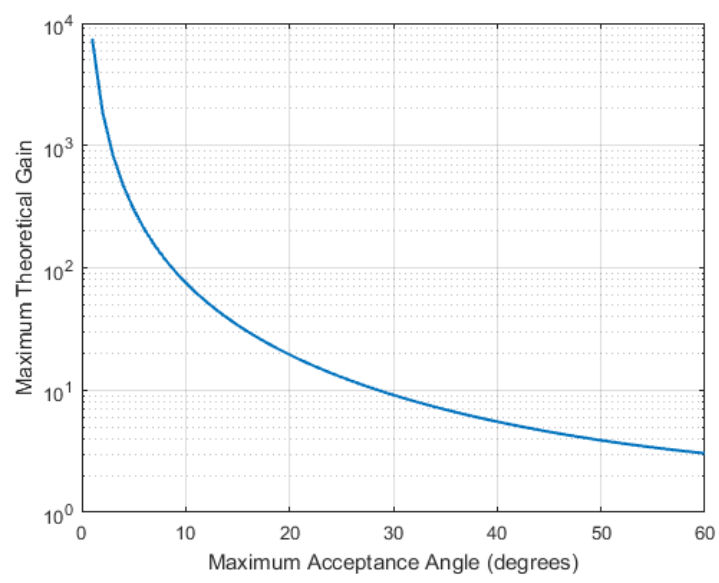

Figure 3. Relation between gain and FOV of a CPC that is made from dielectric material $(n=1.51)$

\subsection{Fluorescent Concentrator (FC)}

The key physical processes in the FC are shown in Figure 4.a. The light that enters the FC will be absorbed by a fluorophore. Some of this light will be re-emitted and retained within the concentrator by total internal reflection (TIR). In a well-designed FC, any light which avoids reabsorption eventually reaches a detector at one end of the FC. These processes therefore make it possible to collect light over a large area and FOV and concentrate it onto a smaller area to achieve high gain.

The gain performance of the FC $\left(G_{C}\right)$ can be calculated from the combination of geometrical gain $\left(G_{A}\right)$ with the probability that a photon will enter the slab, be converted to another wavelength, and reach the detector. If the probability of incident light is reflected by the concentrator surface is $R$, the probability of an incident photon being absorbed is $A$, the quantum yield is $\eta_{P L}$, and the probability of an emitted photon reaching the detector is $\eta_{T I R}$, the concentrating factor can be written as ${ }^{4}$ :

$$
G_{C}=(1-R) \times A \times \eta_{P L} \times \eta_{T I R} \times G_{A}
$$

(a)

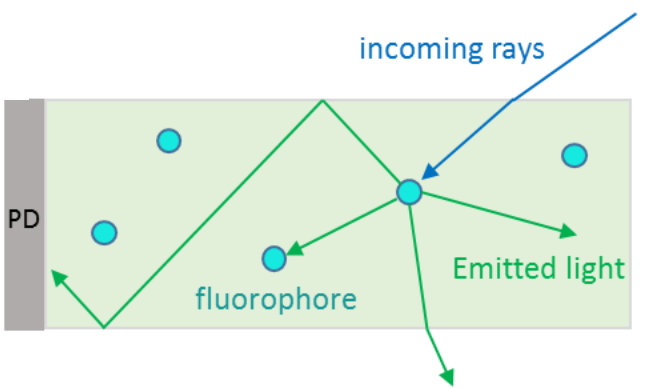

(b)

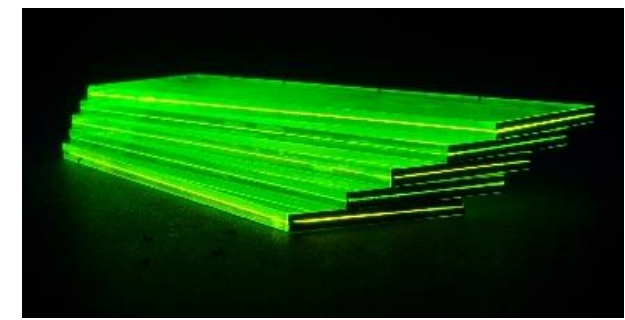

Figure 4. (a) A schematic diagram of the physical processes in a fluorescent concentrator; (b) A photograph of fluorescent concentrators containing Coumarin-6.

\section{EXPERIMENTAL SETUP}

In order to compare the performance of the CPC and FC, a single channel VLC link was built using a commercial LED and detector. Three performance metrics were investigated for CPC and FC, namely: FOV, gain, and received signal quality, as measured using an eye diagram. The experimental setup is shown in Figure 5.

At the transmitter a Blue LED (LED450, Roithner) was driven by a pseudo random binary sequence at $0.3 \mathrm{~mW}$ power using an arbitrary waveform generator (81150A, Keysight). At the receiver, the concentrators were 
installed in a rotating stage where a Power Meter (1830-C, Newport) was used as the detector and coupled to the output aperture of the concentrators. The received signal from the detector was then captured using an Oscilloscope (MS07104B, Keysight), and further signal processing was performed offline. The distance between the transmitter and the receiver was $60 \mathrm{~cm}$.

The CPC measurements were carried out using two samples of commercially available CPCs, namely CPC-65-442 with $\theta_{\max }=25^{\circ}$ and CPC-65-444 with $\theta_{\max }=45^{\circ}$ (Edmund Optics). In this paper, the CPC with $\theta_{\max }=25^{\circ}$ and $\theta_{\text {max }}=45^{\circ}$ will be referred as $\mathrm{CPC}-1$ and $\mathrm{CPC}-2$, respectively.

The FC was fabricated using a $100 \mu \mathrm{m}$ thin fluorescent layer made from Coumarin-6 (Cm6) dye embedded in UV curable epoxy NOA68 ( $\mathrm{n}=1.54)$. Cm6 is selected as the fluorophore dye because of its high quantum yield, absorption peaking in the blue region of the spectrum, and short fluorescence lifetime ${ }^{5}$. The Cm6 layer was sandwiched between two microscope slides ( $25 \mathrm{~mm} \times 75 \mathrm{~mm} \times 1 \mathrm{~mm}, \mathrm{n}=1.52)$ as shown in Figure 4.b.

The FOV measurement was carried out by rotating the receiver through an angle of $0^{\circ}$ to $60^{\circ}$ and observing the received power degradation as the link angle increases. In the eye-diagram measurement, the transmitter was continuously transmitting a pseudorandom binary sequence at $400 \mathrm{~kb} / \mathrm{s}$, which was chosen to minimise the impact of low bandwidth of the source on the measurement. The eye-diagrams from each concentrator sample at various angles were then observed.

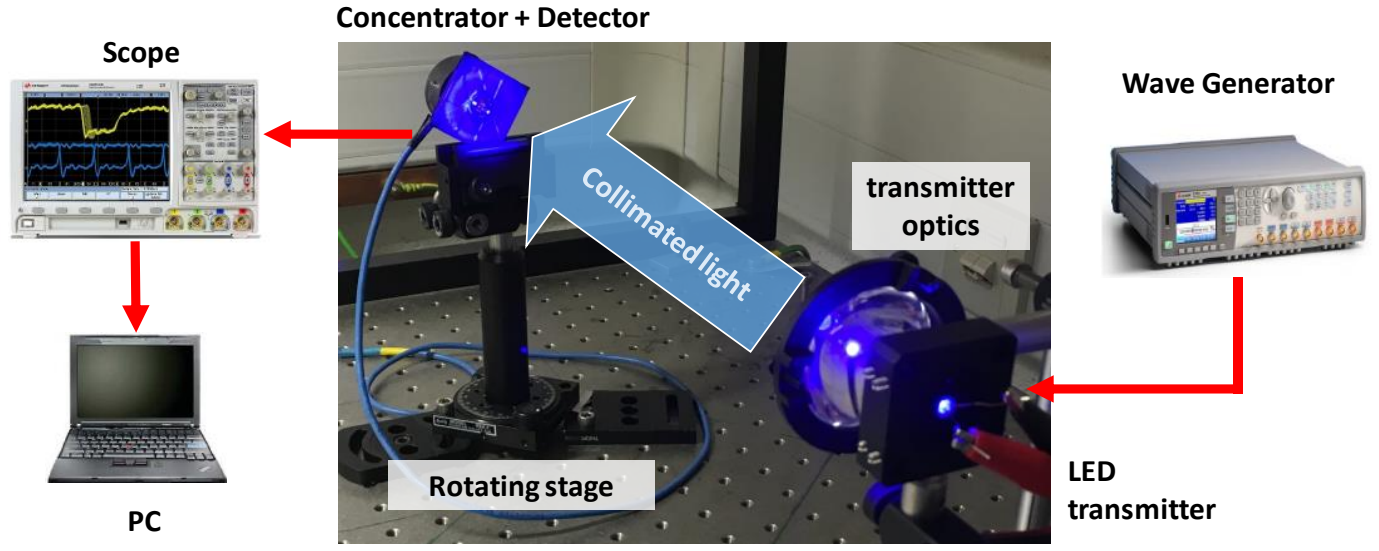

Figure 5. Experimental setup of CPC (shown) and FC measurement. In FC case the detector is positioned on the side.

\section{RESULTS AND DISCUSSION}

The results from the FOV measurement are shown in Figure 6.a. CPC-1 and CPC-2 show minimum received power at $25^{\circ}$ and $45^{\circ}$, which are consistent with their respective specifications. The power degradation of the FC is significantly slower, reaching only half the power at approximately $60^{\circ}$. For comparison, the angle where the power is halved ( $3 \mathrm{~dB}-$ FOV) for CPC-1 and CPC-2 are approximately $20^{\circ}$ and $30^{\circ}$, respectively.

It can be observed in Figure 6.a. that the power degradation of the FC due to increasing angle is similar to the cosine law, due to the rectangular slab form factor of the FC. It can also be observed that the received power in CPC-1 and CPC-2 cases start to drop when the incident angle is increased. This is because the collimated light is larger than the diameter of the CPC input aperture, and as the angle increases, the projected input aperture of both CPC becomes smaller as seen from the transmitter. The power degradation also becomes faster for CPC-1 and CPC- 2 when the incident angle is larger their respective $3 \mathrm{~dB}-\mathrm{FOV}$.

The eye-diagram results (Figure 6.b) show a pattern consistent with the FOV results. At $20^{\circ}$, the amplitude of the eye of $\mathrm{CPC}-1$ is already halved the amplitude and at $45^{\circ}$ there is no clear eye. The amplitude of the eye for CPC- 2 at $20^{\circ}$ is more than half the amplitude at $0^{\circ}$ and at $45^{\circ}$ the eye is almost closed. As expected, the FC shows that its performance degradation is significantly less compared to both CPC-1 and CPC-2, where at $45^{\circ}$ the eye amplitude is still more than half the amplitude at $0^{\circ}$. 
(a)

(b)

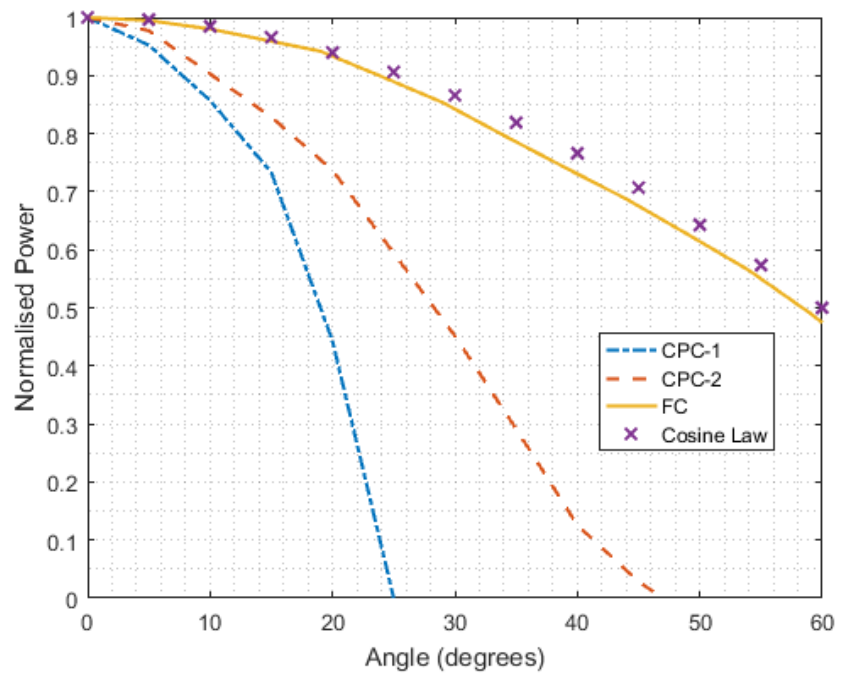

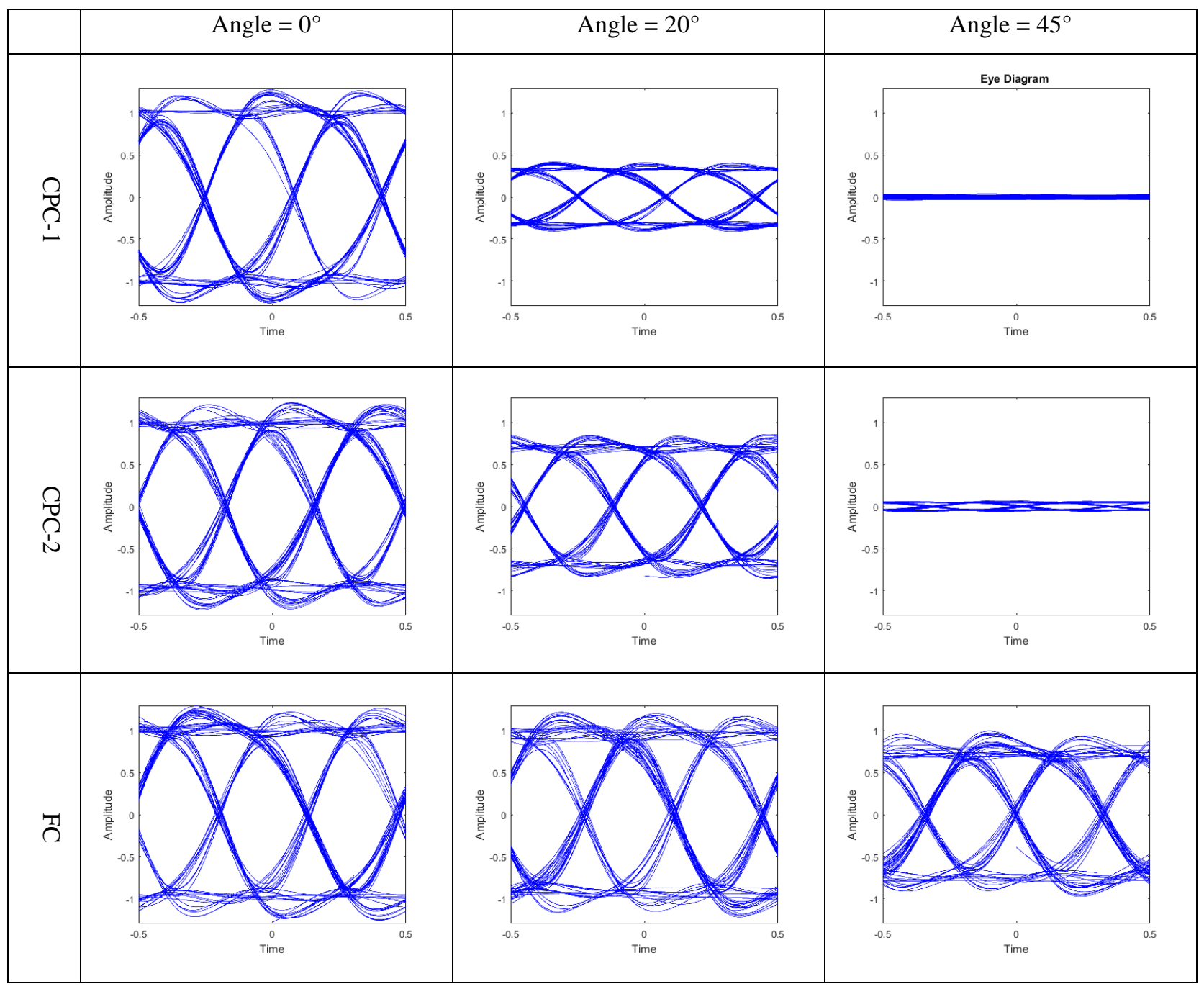

Figure 6. Performance comparison of CPC-1 $\left(\theta_{\max }=25^{\circ}\right), \mathrm{CPC}-2\left(\theta_{\max }=45^{\circ}\right)$, and $\mathrm{FC}$ at various angle of incidence: (a) FOV; (b) Eye-diagram. 
Both CPC-1 and CPC-2 follow the pattern of the relation between maximum gain and FOV as previously depicted in Figure 3, which is gain of 12.7 for CPC- $1\left(\theta_{\max }=25^{\circ}\right)$ and gain of 4.5 for CPC-2 $\left(\theta_{\max }=45^{\circ}\right)$. On the other hand, the FC made from Coumarin-6 sample has a gain of 12 as previously reported ${ }^{6}$. This suggested that FC can achieve similar gain to CPC-1 while having substantially larger FOV. The gain of FC is not limited by étendue but rather by the light loss from escaping light in the edges and the self-absorbance of the emitted light by the fluorophore. To conclude, a comparative summary between the $\mathrm{CPC}-1, \mathrm{CPC}-2$, and $\mathrm{FC}$ is given in Table 1.

Table 1. Comparative summary of optical concentrators for VLC

\begin{tabular}{|c|c|c|c|}
\hline Parameter $\backslash$ Sample & CPC- $1\left(\theta_{\max }=25^{\circ}\right)$ & $\mathrm{CPC}-2\left(\theta_{\max }=45^{\circ}\right)$ & FC \\
\hline Constrained by étendue conservation & Yes & Yes & Lo \\
\hline 3dB-Field-of-View & Small & Medium & High \\
\hline Optical Gain & High & Low & \\
\hline
\end{tabular}

\section{CONCLUSION}

The growing interest in Visible Light Communications (VLC) will increase the needs for a light collector with high gain and wide FOV. Fluorescent Concentrators (FC) might be a potential candidate to fulfil this need. This paper studied the field-of-view (FOV) and optical gain performance of a FC that does not follow classical étendue conservation. A comparison between the FC and conventional optical concentrators, namely Compound Parabolic Concentrator (CPC) was investigated. The trade-off between received signal strength and incoming link angle was demonstrated over a link angle from $0^{\circ}$ to $60^{\circ}$. Future work will focus on material optimisation to minimise the light loss of the FC and the implementation of receivers able to support greater numbers of spatial channels.

\section{ACKNOWLEDGEMENTS}

The research of the first author was funded by the Indonesia Endowment Fund for Education (LPDP). The authors gratefully acknowledge support by the UK Engineering and Physical Sciences Research Council (EPSRC) under grant $\mathrm{EP} / \mathrm{K} 00042 \mathrm{X} / 1$.

\section{REFERENCES}

[1] Elgala, H., Mesleh, R., Haas, H., "Indoor optical wireless communication: potential and state-of-the-art," IEEE Commun. Mag. 49(9), 56-62 (2011).

[2] Steigerwald, D. A., Bhat, J. C., Collins, D., Fletcher, R. M., Holcomb, M. O., Ludowise, M. J., Martin, P. S., Rudaz, S. L., "Illumination with solid state lighting technology," IEEE J. Sel. Top. Quantum Electron. 8(2), 310 320 (2002).

[3] Winston, R., Minano, J. C.., Benitez, P., Nonimaging Optics, 1st ed., Academic Press (2005).

[4] Collins, S., O’Brien, D. C.., Watt, A., "High gain, wide field of view concentrator for optical communications," Opt. Lett. 39(7), 1756 (2014).

[5] Collins, S., Mulyawan, R., Rajbhandari, S., Chu, H., Faulkner, G. E., O’Brien, D. C., Manousiadis, P. P., Vithanage, D. A., Turnbull, G. A., et al., "A simple wide field of view concentrator for free space visible light communications,” 2015 IEEE Summer Top. Meet. Ser., 43-44, IEEE (2015).

[6] Manousiadis, P. P., Rajbhandari, S., Mulyawan, R., Vithanage, D. A., Chun, H., Faulkner, G., O’Brien, D. C., Turnbull, G. A., Collins, S., et al., "Wide field-of-view fluorescent antenna for visible light communications beyond the étendue limit," Optica 3(7), 702 (2016).

[7] Peyronel, T., Quirk, K. J., Wang, S. C.., Tiecke, T. G., "Luminescent detector for free-space optical communication," Optica 3(7), 787 (2016).

[8] Li, G., Su, Y., Pei, G., Yu, X., Ji, J.., Riffat, S., "Preliminary Experimental Comparison of the Performance of a Novel Lens-Walled Compound Parabolic Concentrator (CPC) with the Conventional Mirror and Solid CPCs," Int. J. Green Energy 10(8), 848-859 (2013). 